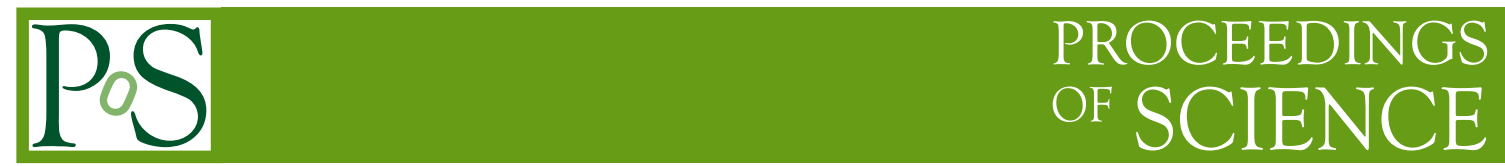

\title{
Charmless B Decays at BABAR and Belle
}

\author{
Simon Sitt (on behalf of the BABAR Collaboration)* \\ LPNHE Paris \\ E-mail: sitteslac.stanford.edu
}

\begin{abstract}
We present recent results from the $B A B A R$ and Belle experiments on charmless hadronic $\mathrm{B}$ decays. The first part is dedicated to hadronic 2-body and quasi-2-body decays and presents the analyses of $\mathrm{B} \rightarrow \mathrm{K}^{+} \pi^{-} \mathrm{K}^{ \pm} \pi^{ \pm}, \mathrm{B} \rightarrow \pi^{+} \pi^{-} \mathrm{K}^{ \pm} \pi^{ \pm}$and $\mathrm{B} \rightarrow \mathrm{X}_{\mathrm{s}} \eta^{(\prime)}$ decays by Belle and of $\mathrm{B} \rightarrow \eta^{\prime} \rho, \eta^{\prime} \mathrm{f}_{0}(980), \eta^{\prime} \mathrm{K}^{*}$ decays by BABAR. The second part is dedicated to hadronic 3-body decays and presents the BABAR analyses of $\mathrm{B}^{0} \rightarrow \mathrm{K}_{\mathrm{S}}^{0} \mathrm{~K}^{ \pm} \pi^{ \pm}, \mathrm{B}^{+} \rightarrow \mathrm{K}^{+} \pi^{0} \pi^{0}$ and $\mathrm{B}^{0} \rightarrow \mathrm{K}_{\mathrm{S}}^{0} \mathrm{~K}_{\mathrm{S}}^{0} \mathrm{~K}_{\mathrm{S}}^{0}$ decays.
\end{abstract}

Flavor Physics and CP Violation - FPCP 2010

May 25-29, 2010

Turin, Italy

\footnotetext{
* Speaker.
} 


\section{Introduction}

Charmless hadronic decays of B mesons can include contributions from both tree level and loop level (or penguin) processes. The penguin processes may have virtual contributions from New Physics that could manifest itself, for instance, by enhanced branching fractions, anomalous CP asymmetries or anomalous polarizations. By studying the interference of light hadron intermediate states that contribute to these decays, informations of the nature of these states can be extracted.

\section{Recent results in charmless hadronic 2-body and quasi-2-body B decays}

\section{1 $\mathrm{B} \rightarrow \mathrm{h}^{+} \pi^{-} \mathrm{K}^{ \pm} \pi^{ \pm}$decays}

The decays $\mathrm{B} \rightarrow \mathrm{K}^{+} \pi^{-} \mathrm{K}^{ \pm} \pi^{ \pm}$and $\mathrm{B} \rightarrow \pi^{+} \pi^{-} \mathrm{K}^{ \pm} \pi^{ \pm}$have contributions of electroweak and gluonic penguins and could help explain why an unexpected low polarization has been measured in $\mathrm{B}$ decays to vector-vector final states, for instance in $\mathrm{B} \rightarrow \phi \mathrm{K}^{*}$, where the polarization was expected close to one, but has been measured to be approximately one half [1][2]. An angular analysis of $\mathrm{B} \rightarrow \mathrm{K}^{+} \pi^{-} \mathrm{K}^{ \pm} \pi^{ \pm}$decays may help clarify if a form factor is at the origin of the low polarization [3]. Additionally this mode can help constraining $\alpha$ and $\gamma$ via the extraction of the longitudinal helicity final state of the resonant mode $\mathrm{B} \rightarrow \mathrm{K}^{*} \overline{\mathrm{K}}^{*}$ that can give insight in the hadronic parameters of $\mathrm{B}_{\mathrm{S}} \rightarrow \mathrm{K}^{*} \overline{\mathrm{K}}^{*}$ decays. An analysis of $\mathrm{B} \rightarrow \pi^{+} \pi^{-} \mathrm{K}^{ \pm} \pi^{ \pm}$decays could give insight into the $\mathrm{K} \pi$-puzzle [4][5], as the difference between the $\mathrm{K} \pi$-modes and their vector-vector counterparts, such as $\mathrm{B} \rightarrow \rho \mathrm{K}^{*}$, is mainly hadronic [6][7]. Furthermore the nonresonant background in vectorvector production that has not yet been studied and could possibly have different properties than the resonant modes.

The Belle analysis of $\mathrm{B} \rightarrow \mathrm{K}^{+} \pi^{-} \mathrm{K}^{ \pm} \pi^{ \pm}$decays [11] does not find a statistically significant signal for $\mathrm{B}^{0} \rightarrow \mathrm{K}^{*} \overline{\mathrm{K}}^{*}$ and $\mathrm{B}^{0} \rightarrow \mathrm{K}^{*} \mathrm{~K}^{*}$ and estimates $90 \%$ C.L. upper limits of $0.8 \times 10^{-6}$ and $0.2 \times 10^{-6}$ respecively. These results are in agreement with the theoretical prediction of $(0.17-$ $0.92) \times 10^{-6}[8]$ and $(2.9 \pm 0.2) \times 10^{-9}$ [9] respectively, but there is a $2.2 \sigma$ discrepancy with the corresponding $B A B A R$ analysis [10] that has measurend a branching fraction of $\left(1.28_{-0.30}^{+0.35}(\mathrm{stat}) \pm\right.$ 0.11 (sys) $) \times 10^{-6}$ for $\mathrm{B}^{0} \rightarrow \mathrm{K}^{*} \overline{\mathrm{K}}^{*}$.

The Belle analysis of $\mathrm{B} \rightarrow \pi^{+} \pi^{-} \mathrm{K}^{ \pm} \pi^{ \pm}$decays [12] makes the first observation of the production mode $\mathrm{B}^{0} \rightarrow \rho^{0} \mathrm{~K}^{+} \pi^{-}$and estimates its branching fraction to be $(2.8 \pm 0.5$ (stat) \pm 0.5 (sys) $) \times$ $10^{-6}$. Belle also finds evidence for $\mathrm{B}^{0} \rightarrow \mathrm{f}_{0}(980) \mathrm{K}^{+} \pi^{-}$and $\mathrm{B}^{0} \rightarrow \pi^{+} \pi^{-} \mathrm{K}^{* 0}$ and measures $(1.4 \pm$ $\left.0.4(\mathrm{stat})_{-0.4}^{+0.3}(\mathrm{sys})\right) \times 10^{-6}$ and $\left(4.5_{-1.0}^{+1.1}(\mathrm{stat})_{-1.6}^{+0.9}(\mathrm{sys})\right) \times 10^{-6}$ respectively. The analysis performs the first measurement of the nonresonant mode but does not find a significant signal, the $90 \%$ C.L. upper limit on the branching fraction is $2.1 \times 10^{-6}$.

\section{2 $\mathrm{B} \rightarrow \mathrm{X}_{\mathrm{s}} \eta$ inclusive}

Charmless B decays that involve $\eta$ and $\eta^{\prime}$ exhibit unique properties due to mixing between the underlying pseudoscalar octet and singlet components [13]. While these mixing effects are relatively well understood in exclusive $\mathrm{B} \rightarrow \mathrm{K}^{(*)} \eta^{(\prime)}$ decays, the picture is less clear in $\mathrm{B} \rightarrow \mathrm{X}_{\mathrm{s}} \eta^{(\prime)}, \mathrm{X}_{\mathrm{s}}$ stands for an inclusive state of unit strangeness. CLEO has made the first observation of $\mathrm{B} \rightarrow \mathrm{X}_{\mathrm{s}} \eta^{\prime}$ and measured a larger than expected branching fraction and a rise in the $\mathrm{X}_{\mathrm{s}}$ spectrum at high mass [14]. These findings have later been confirmed by CLEO [15] and BABAR [16]. A possible 
explanation are large contributions from non-perturbative charming penguin amplitudes [17][18]. A measurement of the complementary process $\mathrm{B} \rightarrow \mathrm{X}_{\mathrm{s}} \eta$ can help to clarify the situation as $\eta-\eta^{\prime}$ mixing suggests that mechanisms with couplings to the pseudoscalar singlet, $\eta^{0}$, should be significant in the $\eta^{\prime}$ mode but less so in the mode with an $\eta$. CLEO previously searched for $\mathrm{B} \rightarrow \mathrm{X}_{\mathrm{s}} \eta$ decays using $3.3 \times 10^{6} \mathrm{~B} \overline{\mathrm{B}}$ pairs, but was only able to place an upper limit on the branching fraction of $4.4 \times 10^{4}$ [14].

The Belle analysis of $\mathrm{B} \rightarrow \mathrm{X}_{\mathrm{s}} \eta$ [24] uses $657 \times 10^{6} \mathrm{~B} \overline{\mathrm{B}}$ pairs and a pseudo-inclusive method for analysis. Belle measures a branching fraction of $(25.5 \pm 2.7$ (stat $) \pm 1.6(\text { syst })_{-14.1}^{+3.8}($ model $\left.)\right) \times 10^{-5}$ for $\mathrm{X}_{\mathrm{s}}$ masses between $0.4 \mathrm{GeV} / c^{2}$ and $2.6 \mathrm{GeV} / c^{2}$, where the systematic error is dominated by the uncertainty of the fragmetation at high mass simulated using PYTHIA and the model uncertainty takes into account contributions that may have been wrongly estimated by the pseudo-inclusive method. This first observation indicates that the mode is not strongly suppressed with respect to $\mathrm{B} \rightarrow \mathrm{X}_{\mathrm{s}} \eta^{\prime}$ and the explanation using charming penguin amplitudes is disfavored. The analysis also confirms the rise at high mass in the $\mathrm{X}_{\mathrm{s}}$ spectrum observed in $\mathrm{B} \rightarrow \mathrm{X}_{\mathrm{s}} \eta^{\prime}$.

\subsection{B decays to $\eta^{\prime} \rho, \eta^{\prime} \mathrm{f}_{0}(980)$ and $\eta^{\prime} \mathrm{K}^{*}$}

There are also pending questions for some exclusive modes involving $\eta^{\prime}$. BABAR has observed $\mathrm{B} \rightarrow \eta^{\prime} \mathrm{K}^{*}$ decays and found evidence for $\mathrm{B} \rightarrow \eta^{\prime} \rho$ analyzing $232 \times 10^{6} \mathrm{~B} \overline{\mathrm{B}}$ pairs. The upper limits reported by Belle [23] using $535 \times 10^{6} \mathrm{~B} \overline{\mathrm{B}}$ pairs are in poor agreement with these results. An update of the $B A B A R$ analysis using more data should be able to clarify the situation. There are theoretical predictions for the branching fractions of these modes, namely from perturbative QCD (pQCD) [19], QCD Factorization (QCDF) [20], Soft Collinear Effective Theory (SCET) [21], and SU (3) flavor symmetry [22]. The predicted branching fractions for the final states $\eta^{\prime} \mathrm{K}^{*}(892)^{+}$ and $\eta^{\prime} \mathrm{K}^{*}(892)^{0}$ are in the range of a few times $10^{6}$, whereas the branching fraction for $\mathrm{B}^{0} \rightarrow \eta^{\prime} \rho^{0}$ is suppressed to $10^{-7}-10^{-8}$. There is some disagreement on the predictions of the branching fraction for $B^{+} \rightarrow \eta^{\prime} \rho^{+}$: SCET calculations give a value of $0.4 \times 10^{-6}$, whereas pQCD and QCDF predict a branching fraction of $(6-9) \times 10^{-6}$. There are no theoretical predictions for the branching fraction of $B^{0} \rightarrow \eta^{\prime} f_{0}(980)$.

The BABAR analysis of $\mathrm{B}$ meson decays to $\eta^{\prime} \rho, \eta^{\prime} \mathrm{f}_{0}(980)$ and $\eta^{\prime} \mathrm{K}^{*}$ [25], where $\mathrm{K}^{*}$ stands for a vector, scalar, or tensor strange meson, studies the decays of $467 \times 10^{6} \mathrm{~B} \overline{\mathrm{B}}$ pairs. BABAR observes significant signal or evidence for $\eta^{\prime} \rho^{+}$and all the $\eta^{\prime} \mathrm{K}^{*}$ channels. The charge asymmetries, where applicable, have also been measured and the results are consistent with no direct CP-violation in all cases. A selection of the results is summarized in Tab. 1. The BABAR results favor the theoretical predictions from perturbative QCD and QCD Factorization and an unexpected enhancement of the tensor $\mathrm{K}_{2}^{*}(1430)$ with respect to the vector $\mathrm{K}^{*}(892)$ component is being observed. The results remain in poor agreement with the results reported by Belle.

\section{Recent results in charmless hadronic 3-body $B$ decays}

3.1 $\mathrm{B}^{0} \rightarrow \mathrm{K}_{\mathrm{S}}^{0} \mathrm{~K}^{ \pm} \pi^{ \pm}$

The decay $\mathrm{B}^{0} \rightarrow \mathrm{K}_{\mathrm{S}}^{0} \mathrm{~K}^{ \pm} \pi^{ \pm}$is suppressed in the Standard Model (even number of Kaons in the final state) and proceeds mainly through $\mathrm{b} \rightarrow \mathrm{u}$ tree and $\mathrm{b} \rightarrow \mathrm{d}$ penguin amplitudes. While there 
Table 1: Statistical significance in units of standard deviations, branching fraction, upper limit on branching fraction, charge asymmetry (if applicable) and the corresponding Belle measurement taken from Reference [23] (if available) for a selection of the analyzed modes. The first errors are statistical and the second errors are systematic uncertainties.

\begin{tabular}{lccccc}
\hline \hline Mode & $\mathrm{S}[\sigma]$ & $\mathrm{BF}\left[10^{-6}\right]$ & $\mathrm{UL} \mathrm{BF}\left[10^{-6}\right]$ & $\mathrm{A}_{\mathrm{ch}}$ & Belle $\left[10^{-6}\right]$ \\
\hline $\mathrm{B} \rightarrow \eta^{\prime} \rho^{0}$ & 2.0 & $1.5 \pm 0.8 \pm 0.3$ & 2.8 & - & $<3.1$ \\
\hline $\mathrm{B} \rightarrow \eta^{\prime} \mathrm{f}_{0}(980)$ & 0.5 & $0.2_{-0.3}^{+0.4} \pm 0.1$ & 0.9 & - & - \\
\hline $\mathrm{B} \rightarrow \eta^{\prime} \rho^{+}$ & $\mathbf{5 . 8}$ & $9.7_{-1.8}^{+1.9} \pm 1.1$ & - & $0.26 \pm 0.17 \pm 0.02$ & $<5.8$ \\
\hline $\mathrm{B} \rightarrow \eta^{\prime} \mathrm{K}^{* 0}$ & 4.0 & $3.1_{-0.8}^{+0.9} \pm 0.3$ & 4.4 & $0.02 \pm 0.23 \pm 0.02$ & $<2.6$ \\
\hline $\mathrm{B} \rightarrow \eta^{\prime} \mathrm{K}^{*+}$ & 3.8 & $4.8_{-1.4}^{+1.6} \pm 0.8$ & 7.2 & $-0.26 \pm 0.27 \pm 0.02$ & $<2.9$ \\
\hline $\mathrm{B} \rightarrow \eta^{\prime}(\mathrm{K} \pi)_{0}^{* 0}$ & $\mathbf{5 . 6}$ & $7.4_{-1.4}^{+1.5} \pm 0.6$ & - & $-0.19 \pm 0.17 \pm 0.02$ & - \\
\hline $\mathrm{B} \rightarrow \eta^{\prime}(\mathrm{K} \pi)_{0}^{*+}$ & 2.9 & $6.0_{-2.0}^{+2.2} \pm 0.9$ & 9.3 & $0.06 \pm 0.20 \pm 0.02$ & - \\
\hline $\mathrm{B} \rightarrow \eta^{\prime} \mathrm{K}_{2}^{*}(1430)^{0}$ & $\mathbf{5 . 3}$ & $13.7_{-2.9}^{+3.0} \pm 1.2$ & - & $0.14 \pm 0.18 \pm 0.02$ & - \\
\hline $\mathrm{B} \rightarrow \eta^{\prime} \mathrm{K}_{2}^{*}(1430)^{+}$ & $\mathbf{7 . 2}$ & $28.0_{-4.3}^{+4.6} \pm 2.6$ & - & $0.15 \pm 0.13 \pm 0.02$ & - \\
\hline \hline
\end{tabular}

have been observations of $B^{0} \rightarrow K_{S}^{0} K_{S}^{0}$ and $B^{+} \rightarrow K_{S}^{0} K^{+}$[26][27] and the related vector-vector final states have also been detected [28][29] there exist only upper limits for the corresponding pseudoscalar-vector final states: $\mathscr{B}\left(\mathrm{B}^{0} \rightarrow \mathrm{K}^{0} \overline{\mathrm{K}}^{* 0}\right)+\mathscr{B}\left(\mathrm{B}^{0} \rightarrow \overline{\mathrm{K}}^{0} \mathrm{~K}^{* 0}\right)<1.9 \times 10^{-6}$ [30] and $\mathscr{B}\left(\mathrm{B}^{+} \rightarrow \mathrm{K}^{+} \overline{\mathrm{K}}^{* 0}\right)<1.1 \times 10^{-6}$ [31]. An analysis of $\mathrm{B}^{0} \rightarrow \mathrm{K}_{\mathrm{S}}^{0} \mathrm{~K}^{ \pm} \pi^{ \pm}$decays could also help to clarify the nature of the so-called $\mathrm{f}_{\mathrm{X}}(1500)$ resonance of unknown spin and isospin quantum numbers that has been observed in $\mathrm{B}^{+} \rightarrow \mathrm{K}^{+} \mathrm{K}^{-} \pi^{+}$[32] but not in $\mathrm{B}^{+} \rightarrow \mathrm{K}_{\mathrm{s}}^{0} \mathrm{~K}_{\mathrm{s}}^{0} \pi^{+}$[33], by searching for an isospin partner to the $\mathrm{f}_{\mathrm{X}}(1500)$ that decays to $\overline{\mathrm{K}}^{0} \mathrm{~K}^{+}$.

The BABAR analysis of $\mathrm{B}^{0} \rightarrow \mathrm{K}_{\mathrm{S}}^{0} \mathrm{~K}^{ \pm} \pi^{ \pm}$[34] uses the decays of $465 \times 10^{6} \mathrm{~B} \overline{\mathrm{B}}$ pairs and make the first observation of the decay mode. The measured branching fraction is $\mathscr{B}\left(B^{0} \rightarrow K_{S}^{0} K^{ \pm} \pi^{ \pm}\right)=$ $(3.2 \pm 0.5($ stat $) \pm 0.3($ sys $)) \times 10^{-6}$ and has been obtained using the ${ }_{s} \mathscr{P}$ lots technique [35] to estimate the efficiency-corrected signal distribution over the Dalitz plot. Quantitative statements concerning the content of the Dalitz plot require a dedicated amplitude analysis. However it appears that there is no major contribution from an isospin partner of the $\mathrm{f}_{\mathrm{X}}(1500)$ decaying to $K_{S}^{0} K^{+}$ , which contrasts to the clear signal seen in $\mathrm{B}^{+} \rightarrow \mathrm{K}^{+} \mathrm{K}^{-} \pi^{+}$decays [32].

\subsection{Observation of the rare decay $\mathrm{B}^{+} \rightarrow \mathrm{K}^{+} \pi^{0} \pi^{0}$}

The decay $\mathrm{B}^{+} \rightarrow \mathrm{K}^{*+} \pi^{0}$ has currently the largest experimental uncertainty of all $\mathrm{B} \rightarrow \mathrm{K}^{*} \pi$ decays. This pseudoscalar-vector decay could help to understand the puzzle in the related $\mathrm{K} \pi$ decays [4][5], where the measured branching fractions and $C P$ violation pattern are incompatible with the naive Standard Model expectation. Furthermore information on the Dalitz plot distribution may also help to clarify the interpretation of the inclusive time-dependent analyses of $\mathrm{B}^{0} \rightarrow \mathrm{K}_{\mathrm{s}}^{0} \pi^{0} \pi^{0}[36][37]$.

The preliminary BABAR analysis of $\mathrm{B}^{+} \rightarrow \mathrm{K}^{+} \pi^{0} \pi^{0}$ decays [38] uses $471 \times 10^{6} \mathrm{~B} \overline{\mathrm{B}}$ events and observes an excess of signal with a significance above 10 standard deviations including systematic 
uncertainties and measures the branching fraction to be $\mathscr{B}\left(\mathrm{B}^{+} \rightarrow \mathrm{K}^{+} \pi^{0} \pi^{0}\right)=(15.5 \pm 1.1$ (stat $) \pm$ $1.6($ sys $)) \times 10^{-6}$. The largest systematic uncertainties come from Probability Density Function uncertainties and the uncertainty of the $\pi^{0}$ reconstruction efficiency. The analysis is a first step towards understanding the Dalitz plot structure.

\subsection{Amplitude analysis of $\mathrm{B}^{0} \rightarrow \mathrm{K}_{\mathrm{S}}^{0} \mathrm{~K}_{\mathrm{S}}^{0} \mathrm{~K}_{\mathrm{S}}^{0}$ decays}

The decay mode $\mathrm{B}^{0} \rightarrow \mathrm{K}_{\mathrm{S}}^{0} \mathrm{~K}_{\mathrm{S}}^{0} \mathrm{~K}_{\mathrm{S}}^{0}$ is of particular interest for time-dependent analysis, as theoretical uncertainties are very small [39] and it is experimentally very clean. Furthermore the time-dependent $C P$ violation parameters $\mathrm{S}$ and $\mathrm{C}$ can be extracted inclusively, as the final state is $C P$ definite[40]. Time-dependent analyses have been be performed by BABAR [41] and Belle [42]. Nevertheless there is no reason why the quasi-2-body parameters should be the same for all intermediate states (that are also $C P$ definite) and an amplitude analysis would give insight into the resonant content of the decay. Another particularity of the decay is that, due to angular momentum conservation, only even-spin intermediate states are permitted. Hence an observation of the $\mathrm{f}_{\mathrm{X}}(1500)$ resonance (compare to $\mathrm{B}^{0} \rightarrow \mathrm{K}_{\mathrm{S}}^{0} \mathrm{~K}^{ \pm} \pi^{ \pm}$analysis above), that has also been observed in $\mathrm{B}^{0} \rightarrow \mathrm{K}^{+} \mathrm{K}^{-} \mathrm{K}_{\mathrm{s}}^{0}$ and $\mathrm{B}^{ \pm} \rightarrow \mathrm{K}^{+} \mathrm{K}^{-} \mathrm{K}^{ \pm}$decays by BABAR [43][44] and Belle [45], would make a scalar nature more likely.

The preliminary BABAR amplitude analysis of $\mathrm{B}^{0} \rightarrow \mathrm{K}_{\mathrm{S}}^{0} \mathrm{~K}_{\mathrm{S}}^{0} \mathrm{~K}_{\mathrm{S}}^{0}$ decays [46] uses $468 \times 10^{6} \mathrm{~B} \overline{\mathrm{B}}$ pairs. As a time-dependent amplitude analysis is not possible due to the limited statistics, this first amplitude analysis is time-integrated and $C P$ blind. Due to the three identical bosons in the final state, the amplitude, that is described using the isobar model, is completely symmetric and a unique position on the Dalitz plot is defined using the minimum and the maximum of the invariant masses. $B A B A R$ finds contributions from $\mathrm{f}_{0}(980), \mathrm{f}_{0}(1710), \mathrm{f}_{2}(2010)$ and nonresonant decays and measures the product branching fractions $\left(2.69_{-1.18}^{+1.24} \pm 0.36 \pm 1.87\right) \times 10^{-6},\left(0.50_{-0.23}^{+0.46} \pm 0.04 \pm 0.13\right) \times 10^{-6}$, $\left(0.54_{-0.20}^{+0.21} \pm 0.03 \pm 0.44\right) \times 10^{-6}$ and $\left(13.31_{-2.30}^{+2.23} \pm 0.55 \pm 2.77\right) \times 10^{-6}$ respectively. BABAR also measures the total inclusive branching fraction $\mathscr{B}\left(\mathrm{B}^{0} \rightarrow \mathrm{K}_{\mathrm{S}}^{0} \mathrm{~K}_{\mathrm{S}}^{0} \mathrm{~K}_{\mathrm{s}}^{0}\right)=(6.18 \pm 0.47 \pm 0.14 \pm 0.06) \times$ $10^{-6}$. The first quoted uncertainty on each branching fraction is statistical, the second is systematic and the third corresponds to the Dalitz plot model uncertainties. The model uncertainties are dominated by the poorly measured mass and width of the $f_{2}(2010)$ resonance [47]. There is a second solution in the likelihood fit that is disfavored by almost two standard deviations and the branching fractions have been calculated using the best solution. The isobar fractions and relative phases of all contributions for both solutions are summarized in Tab. 2. The controversial $f_{X}(1500)$ resonance does not need to be included in the signal model to describe the data. The peak in the invariant mass between 1.5 and $1.6 \mathrm{GeV} / c^{2}$ can be described by the interference between the $\mathrm{f}_{0}(1710)$ resonance and the nonresonant component.

\section{Conclusions and perspectives}

With the data accumulated at the $\mathrm{B}$ factories, many rare charmless $\mathrm{B}$ decays are now accessible. The recent analyses of $\mathrm{B} \rightarrow \mathrm{h}^{+} \pi^{-} \mathrm{K}^{ \pm} \pi^{ \pm}$decays add information on the polarization puzzle. Concerning the measurements of $\mathrm{B} \rightarrow \eta^{(/)} \mathrm{X}$ decays, there is some remaining discrepancy between the BABAR and Belle results. Recent results in charmless hadronic 3-body $\mathrm{B}$ decays help to under- 
Table 2: Summary of measurements of isobar fractions (FF) and relative phases. The errors are statistical only. The statistical significance has been estimated from the variation of the likelihood when removing the resonances one by one from the fit (significance $=\sqrt{-2 \Delta \log \mathscr{L}}$ ).

\begin{tabular}{|c|c|c|}
\hline Mode & Solution 1 & Solution 2 \\
\hline $\mathrm{FF} \mathrm{f}_{0}(980) \mathrm{K}_{\mathrm{s}}^{0}$ & $0.44_{-0.19}^{+0.20}$ & $1.03_{-0.17}^{+0.22}$ \\
\hline Phase $[\mathrm{rad}] \mathrm{f}_{0}(980) \mathrm{K}_{\mathrm{s}}^{0}$ & $0.09 \pm 0.16$ & $1.26 \pm 0.17$ \\
\hline Significance $[\sigma] \mathrm{f}_{0}(980) \mathrm{K}_{\mathrm{s}}^{0}$ & 3.3 & - \\
\hline $\mathrm{FF} \mathrm{f}_{0}(1710) \mathrm{K}_{\mathrm{s}}^{0}$ & $0.07_{-0.03}^{+0.07}$ & $0.09_{-0.02}^{+0.05}$ \\
\hline Phase $[\mathrm{rad}] \mathrm{f}_{0}(1710) \mathrm{K}_{\mathrm{s}}^{0}$ & $1.11 \pm 0.23$ & $0.36 \pm 0.20$ \\
\hline Significance $[\sigma] \mathrm{f}_{0}(1710) \mathrm{K}_{\mathrm{s}}^{0}$ & 3.7 & - \\
\hline $\mathrm{FF} \mathrm{f}_{2}(2010) \mathrm{K}_{\mathrm{s}}^{0}$ & $0.09_{-0.03}^{+0.03}$ & $0.10 \pm 0.02$ \\
\hline Phase $[\mathrm{rad}] \mathrm{f}_{2}(2010) \mathrm{K}_{\mathrm{s}}^{0}$ & $2.50 \pm 0.20$ & $1.58 \pm 0.22$ \\
\hline Significance $[\sigma] \mathrm{f}_{2}(2010) \mathrm{K}_{\mathrm{s}}^{0}$ & 3.3 & - \\
\hline $\mathrm{FF} \chi_{\mathrm{c} 0} \mathrm{~K}_{\mathrm{s}}^{0}$ & $0.07_{-0.02}^{+0.04}$ & $0.07 \pm 0.02$ \\
\hline Phase $[\mathrm{rad}] \chi_{\mathrm{c} 0} \mathrm{~K}_{\mathrm{s}}^{0}$ & $0.63 \pm 0.47$ & $-0.24 \pm 0.52$ \\
\hline Significance $[\sigma] \chi_{\mathrm{c} 0} \mathrm{~K}_{\mathrm{s}}^{0}$ & 4.2 & - \\
\hline FF NR & $2.15_{-0.37}^{+0.36}$ & $1.37_{-0.21}^{+0.26}$ \\
\hline Phase [rad] NR & 0.0 & 0.0 \\
\hline Significance $[\sigma] \mathrm{NR}$ & 8.2 & - \\
\hline Total FF & $2.84_{-0.66}^{+0.71}$ & $2.66_{-0.27}^{+0.35}$ \\
\hline
\end{tabular}

stand the nature of the controversial $f_{X}(1500)$ resonance. All the presented results are statistically limited and would benefit from next generation B factories. 


\section{References}

[1] A. Ali et al., Z. Phys. C 1, 269 (1979); M. Suzuki, Phys. Rev. D 66, 054018 (2002)

[2] C.H. Chen, Y.Y. Keum, and H-n. Li, Phys. Rev. D 66, 054013 (2002)

[3] H-n. Li, Phys. Lett. B 622, 63 (2005)

[4] S.-W. Lin et al. [Belle Collaboration], Nature 452, 332 (2008)

[5] B. Aubert et al. [BaBar Collaboration], Phys. Rev. Lett. 99, 021603 (2007).

[6] M. Beneke, et al., Phys. Rev. Lett. 96, 141801 (2006)

[7] C. S. Kim et al., Phys. Rev. D 76, 074019 (2007)

[8] W. Zou and Z. Xiao, Phys. Rev. D 72, 094026 (2005); M. Beneke, J. Rohrer, and D. Yang, Nucl. Phys. B774, 64 (2007). H.-Y. Cheng and K.-C. Yang, Phys. Rev. D 78, 094001 (2008)

[9] D. Pirjol and J. Zupan, arXiv:0908.3150 [hep-ph]

[10] B. Aubert [BaBar Collaboration], Phys. Rev. Lett. 100, 081801 (2008).

[11] C.C. Chiang et al. [Belle collaboration], PRD 81, 071101(R) (2010).

[12] S.-H.Kyeong, Y.-J.Kwon et al. [Belle collaboration], PRD 80, 051103 (2009).

[13] H.J. Lipkin, Phys. Lett. B 254, 247 (1991).

[14] T.E. Browder et al. [CLEO Collaboration], Phys. Rev. Lett. 81, 1786 (1998).

[15] G. Bonvicini et al. [CLEO Collaboration], Phys. Rev. D 68, 011101 (2003).

[16] B. Aubert et al. [BaBar Collaboration], Phys. Rev. Lett. 93, 061801 (2004).

[17] I. E. Halperin and A. Zhitnitsky, Phys. Rev. Lett. 80, 438 (1998).

[18] J. Chay, C. Kim, A. K. Leibovich, and J. Zupan, Phys. Rev. D 74, 092005 (2007).

[19] X. Liu et al., Phys. Rev. D 73, 074002 (2006); A. G. Akeroyd, C. H. Chen, and C. Q. Geng, Phys. Rev. D 75, 054003 (2007).

[20] M. Beneke and M. Neubert, Nucl. Phys. B 675, 333 (2003).

[21] W. Wang, Y. M. Wang, D. S. Yang, and C. D. Lu, Phys. Rev. D 78, 034011 (2008).

[22] C.-W. Chiang and Y.-F. Zhou, JHEP 03, 055 (2009).

[23] J. Schümann et al. [Belle Collaboration], Phys. Rev. D 75, 092002 (2007).

[24] K.Nishimura, T.Browder et al. [Belle collaboration], PRL 105, 191803 (2010).

[25] P. del Amo Sanchez et al. [BaBar Collaboration], PRD-RC 82, 011502 (2010).

[26] B. Aubert et al. [BaBar Collaboration], Phys. Rev. Lett. 97 (2006) 171805.

[27] K. Abe et al. [Belle Collaboration], Phys. Rev. Lett. 98 (2007) 181804.

[28] B. Aubert et al. [BaBar Collaboration], Phys. Rev. Lett. 100 (2008) 081801.

[29] B. Aubert et al. [BaBar Collaboration], Phys. Rev. D 79 (2009) 051102.

[30] B. Aubert et al. [BaBar Collaboration], Phys. Rev. D 74 (2006) 072008.

[31] B. Aubert et al. [BaBar Collaboration], Phys. Rev. D 76 (2007) 071103. 
[32] B. Aubert et al. [BaBar Collaboration], Phys. Rev. Lett. 99 (2007) 221801.

[33] B. Aubert et al. [BaBar Collaboration], Phys. Rev. D 79 (2009) 051101.

[34] P. del Amo Sanchez et al. [BaBar Collaboration], PRD-RC 82, 031101 (2010).

[35] Pivk, Muriel and Le Diberder, Francois R., Nucl. Instrum. Meth., A555 (2005), 356-369.

[36] B. Aubert et al. [BaBar Collaboration], Phys. Rev. D76, 071101 (2007), hep-ex/0702010.

[37] K. Abe et al. [Belle Collaboration] (2007), arXiv:0708.1845 [hep-ex].

[38] P. del Amo Sanchez et al. [BaBar Collaboration], arXiv:1005.3717.

[39] H.-Y. Cheng, C.-K. Chua, and A. Soni, Phys. Rev. D72, 094003 (2005).

[40] T. Gershon and M. Hazumi, Phys. Lett. B596, 163 (2004).

[41] B. Aubert et al. [BaBar Collaboration], Phys. Rev. D 76, 091101 (2007).

[42] K.-F. Chen et al. [Belle Collaboration], Phys. Rev. Lett. 98, 031802 (2007).

[43] B. Aubert et al. [BaBar Collaboration], Phys. Rev. D 74, 032003 (2005), 0605003.

[44] B. Aubert et al. [BaBar Collaboration], Phys. Rev. Lett. 99, 161802 (2007).

[45] A. Garmash et al. [Belle Collaboration], Phys. Rev. D 71, 092003 (2005), 0412066.

[46] S. Sitt, Thèse de doctorat, Université Pierre et Marie Curie Paris, unpublished (2010)

[47] C. Amsler et al. [Particle Data Group], Phys. Lett. B667 (2008). 